\title{
Inhibition of Electrochemical Fouling against Biomolecules on a Diamond-Like Carbon Electrode
}

\author{
Takuya Goto, ${ }^{*}$ Tomoyuki Yasukawa, ${ }^{*}, *+$ Kazuhiro Kanda, ${ }^{* * *}$ Shinji Matsui, ${ }^{* * *}$ and \\ Fumio Mizutani*广 \\ *Graduate School of Material Science, University of Hyogo, 3-2-1 Kouto, Kamigori, Ako-gun, \\ Hyogo 678-1297, Japan \\ **JST-CREST, 5 Sanbancho, Chiyoda, Tokyo 102-0075, Japan \\ ***Laboratory of Advanced Science and Technology for Industry, University of Hyogo, 3-1-2 Kouto, Kamigori, \\ Ako-gun, Hyogo 678-1205, Japan
}

\begin{abstract}
An anti-fouling ability of diamond-like carbon (DLC) electrodes to biological macromolecules has been investigated from a decrease in the electrochemical redox current of $\mathrm{Fe}(\mathrm{CN})_{6}{ }^{4-13-}$, used as a redox marker. A DLC electrode and a glassy carbon (GC) electrode were immersed in a solution containing bovine serum albumin (BSA) or DNA. The GCs treated with biological macromolecules gave rise to a significant decrease in the currents, while there was no signal decreases from the treated DLCs. The signals from the DLCs remain essentially unchanged for at least $24 \mathrm{~h} \mathrm{at} \mathrm{a} 10 \mathrm{mg} / \mathrm{mL}$ concentration level of BSA.
\end{abstract}

(Received October 15, 2010; Accepted November 29, 2010; Published January 10, 2011)

\section{Introduction}

Novel carbon materials, such as conducting diamond, diamond-like carbon (DLC), electron cyclotron resonance (ECR)-sputtered nanocrystalline carbon, pyrolyzed photoresist, and fullerens have come into use for electrochemisutry during the last two decades. ${ }^{1}$ DLC is an amorphous carbon with a high fraction of diamond-like $\left(\mathrm{sp}^{3}\right)$ bond, which is hard and wear-resistant, and is used for coating hard-disk drive heads. ${ }^{1,2}$ The high ohmic resistivity of DLCs can be decreased by adding other elements, and also by varying the deposition conditions to prepare the carbon films. The excellent physical properties as well as chemical inertness to acidic and alkaline media and organic solvents ${ }^{3}$ have led us to utilize DLCs as electrode materials in electroanalytical systems..$^{3-6}$

For determining the electroactive species in biological fluids, electrode fouling due to the adsorption of macromolecules, such as proteins and DNA, is a serious problem. The adsorption of macromolecules on carbon electrodes is considered to be caused by various kinds of interactions between the adsorbants and the electrode surfaces. For example, the interactions of macromolecules by hydorogen bonding with the surface oxides, ${ }^{1}$ physicochemical interactions at grain boundaries, and trapping in nm-sized defects would be responsible for the molecular adsorption and for electrode fouling. Hence it seems reasonable that ECR-sputtered nanocrystalline carbon ${ }^{7-9}$ electrodes with a relatively high $\mathrm{sp} 3$ content, which is characterized by a low surface oxide concentration, and grain-boundary free, flat surface, show much higher resistivity to fouling than conventional glassy carbon (GC) electrodes. Boron-doped

† To whom correspondence should be addressed.

E-mail: yasu@ sci.u-hyogo.ac.jp; mizutani@ @sci.u-hyogo.ac.jp diamond (BBD), which mainly consists of $\mathrm{sp}^{3}$ bonds, also exhibited excellent electrochemical properties, such as a wide potential window, low capacitive current and low surface fouling. ${ }^{10}$ The surface properties of DLCs are similar to those of ECR-sputtered nanocrystalline carbon with respect to the low surface oxide concentration and surface flatness without any grain-boundaries. Thus, we can expect that DLCs are also more resistant to fouling than GC electrodes. Actually, higher resistivity against the addition of biomolecules has been proved by our preliminary examinations; the current responses upon the addition of a redox marker $\left(\mathrm{Fe}(\mathrm{CN})_{6}{ }^{4 / 3-}\right.$ system $)$ did not strikingly decreased after soaking a DLC electrode in a bovine serum albumin (BSA), DNA or serum-containing solution, whereas a GC electrode was seriously fouled by such a treatment.

\section{Experimental}

\section{Chemicals}

Tris(2,2'-bipyridyl)dichlororuthenium(II) hexahydrate, potassium ferrocyanide and ferrocene methanol were obtained from Aldrich (Milwaukee, WI). BSA and DNA (from herring sperm, $\leq 50 \mathrm{bp}$ ) were obtained from Sigma (St. Louis, MO). Human sera and fluorescein-attached BSA were purchased from Nissui Pharmaceutical (Tokyo) and Invitrogen Japan K.K. (Tokyo), respectively. Other reagents were of analytical reagent grade (Nacalai Tesque, Kyoto). Distilled and deionized water was used throughout.

\section{Electrochemical measurements}

The conducting DLC plate used was obtained from Nanotec Co. (Chiba). The thickness and electrical resistivity of the DLC films were $500 \mathrm{~nm}$ and $2.5 \Omega \mathrm{m}$, respectively. Cyclic voltammetry 
(a)



(b)

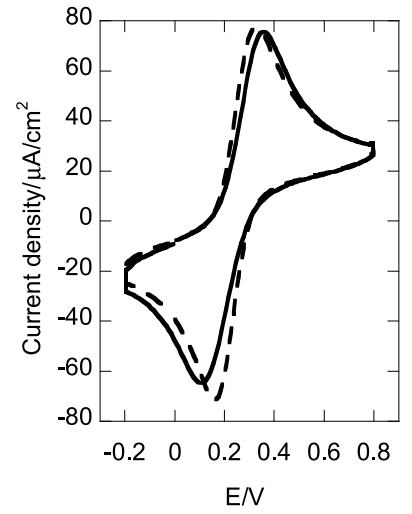

(c)

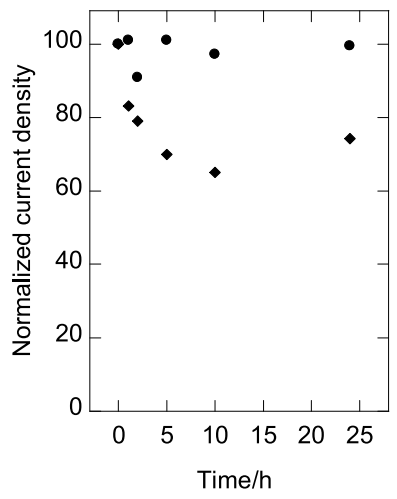

Fig. 1 Cyclic voltammograms for $0.1 \mathrm{mM}$ ferrocyanide obtained on (a) GC and (b) DLC treated with oxygen plasma. Solid curves and dashed curves are before and after treating with $10 \mathrm{mg} / \mathrm{mL}$ BSA for $1 \mathrm{~h}$, respectively. (c) Relationships between the magnitude of the oxidation peak for the $\mathrm{Fe}(\mathrm{CN})_{6}{ }^{4-}$ and the time for the BSA treatment on the GC (diamond) and DLC (circle).

(CV) was performed using an HSV-100 electrochemical analyzer (Hokuto Denko, Tokyo) with a conventional three-electrode system. A glassy carbon $(1 \mathrm{~mm}$ in diameter, BAS, Tokyo) and DLC (4 $\mathrm{mm}$ in diameter) were used as the working electrode, and an $\mathrm{Ag} / \mathrm{AgCl}$ (saturated with $\mathrm{KCl}$ ) and a platinum wire were served as the reference and auxiliary electrodes, respectively. Prior to the CV measurement, the DLC was treated with oxygen plasma for 1 min (Type LTA-302, Yanagimoto Manufacturing, Kyoto) to introduce hydrophilicity on the surface. The GC was also treated with oxygen plasma after polishing with alumina/water slurry and washing with water. It is known that the hydrophilic surface is suppressed due to the adsorption of macromolecules with the ECR-sputtered carbon. $^{11,12}$ The supporting electrolyte solution was a $0.1 \mathrm{M}$ potassium phosphate buffer solution ( $\mathrm{pH}$ 7.0). The solution was saturated with air and kept at room temperature. The potential sweep rate for the CV measurement was usually set at $100 \mathrm{mV} / \mathrm{s}$. Each data was evaluated as the average obtained from five different experiments.

\section{Atomic force microscopy and fluorescence measurements}

Atomic force microscopy (AFM) measurements were carried out with an E-sweep/NanoNavi Station (SII NanoTechnology Inc., Chiba). Images were obtained in the tapping mode at scan rates of $0.3 \mathrm{~Hz}$. Fluorescence microscopy (BZ-8100, Keyence, Osaka) was used to detect fluorescein-attached BSA on surfaces of the GC and DLC plates. Microchannels were prepared with polydimethylsiloxane (PDMS) by the conventional photolithography. The PDMS pre-polymer was poured on a mold of the microchannel $(10 \mathrm{~mm}$ length, $1.0 \mathrm{~mm}$ wide and $50 \mu \mathrm{m}$ height) fabricated by a negative photoresist, SU-8 3050 (MicroChem Corp., Newon, MA). The PDMS microchannel was peeled and mounted on each plate. A solution containing $1.0 \mathrm{mg} / \mathrm{mL}$ fluorescein-attached BSA was introduced into the PDMS channel for $1 \mathrm{~h}$. After peeling off and washing, the surfaces of each plate were observed with fluorescence microscopy.

\section{Results and Discussion}

Basic electrochemical properties of DLC film electrode

We investigated the electrochemical properties of DLC and
GC electrodes that were treated with oxygen plasma by cyclic voltammetry in $0.05 \mathrm{M} \mathrm{H}_{2} \mathrm{SO}_{4}$. The potential window of DLC $(2.4 \mathrm{~V})$ was nearly the same as that of GC $(2.4 \mathrm{~V})$, but shifted by about $0.2 \mathrm{~V}$ to the positive potential region compared to the GC. The potential window is defined as a potential range within $\pm 500 \mu \mathrm{A} / \mathrm{cm}^{2}$ oxidation/reduction current densities. ${ }^{13}$ Furthermore, the much smaller non-faradaic current of DLC (around $<0.1 \mu \mathrm{A} / \mathrm{cm}^{2}$ ), which is attributable to the flat surface structure and low density of the electronic state ${ }^{1}$ of DLC, is observed compared to that of GC (around $1.5 \mu \mathrm{A} / \mathrm{cm}^{2}$ ) in the middle of the potential window. These properties indicate that the DLC electrode used in this work is robust for electrochemical measurements in an aqueous solution compared to the general GC electrodes.

Effect of BSA adsorption on the redox responses for $\mathrm{Fe}(\mathrm{CN})_{6}^{4-13-}$

The solid curves in Figs. 1a and $1 \mathrm{~b}$ show CVs for $0.1 \mathrm{mM}$ ferrocyanide obtained on GC and DLC electrodes, respectively. The CVs on both electrodes were almost similar to each other with respect to the redox potentials and peak heights, although the magnitude of the non-faradaic current for the DLC was much smaller than the GC. This indicates that the electron transfer rate for the $\mathrm{Fe}(\mathrm{CN})_{6}{ }^{4-13-}$ system on the DLC electrode is as large as that on the $\mathrm{GC}$ electrode.

The $\mathrm{CV}$ for the $\mathrm{Fe}(\mathrm{CN})_{6}{ }^{4 / 3-}$ system was recorded after soaking each electrode in a $10 \mathrm{mg} / \mathrm{mL}$ BSA solution for $1 \mathrm{~h}$. The dashed curves in Figs. 1a and 1b denote the results obtained for the GC and DLC. As can been seen in Fig 1a, the BSA treatment has brought about a significant increase in the peak separation (from $187 \pm 3$ to $540 \pm 60 \mathrm{mV}$ ), and a decrease in the peak current (to $82 \pm 6 \%$ of the initial value) for the GC electrode. In contrast, the same treatment did not show a discernible electrode-fouling effect on the DLC. Suppression of the macromolecular adsorption would result in the anti-fouling ability of the electrode. Figure 1c shows the relationships between the magnitude of the oxidation peak for $\mathrm{Fe}(\mathrm{CN})_{6}{ }^{4-}$ and the time for the BSA treatment. The current response on the GC decreased with increasing time for the treatment (diamonds in Fig. 1c), while it on the DLC did not decrease for at least $24 \mathrm{~h}$ (circles in Fig. 1c). Thus, the suppression of BSA adsorption has been proved to bring about the anti-fouling ability of the electrode.

Furthermore, measurements with AFM and a fluorescent microscopy have revealed that the adsorption of BSA on the 


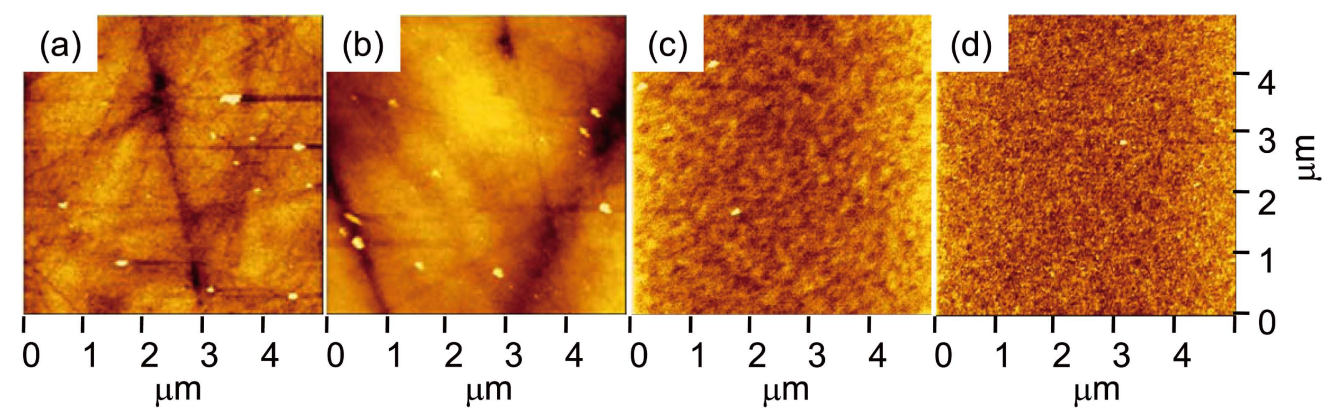

Fig. 2 AFM images of GC (a and b) and DLC (c and d) surfaces before (a and c) and after (b and d) treating with $10 \mathrm{mg} / \mathrm{mL} \mathrm{BSA}$ for $1 \mathrm{~h}$.

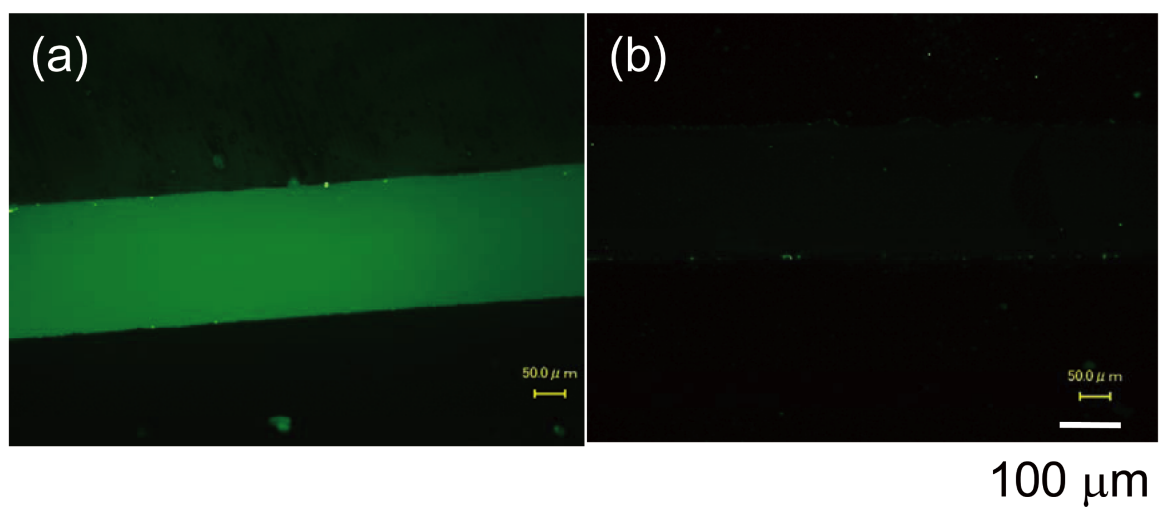

Fig. 3 Fluorescence images of fluorescein-attached BSA adsorbed on (a) GC and (b) DLC plates.

(a)

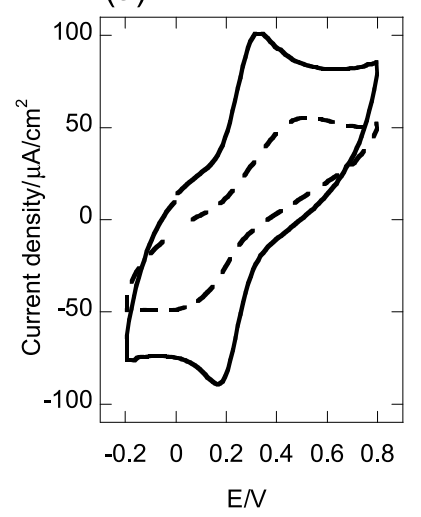

(b)

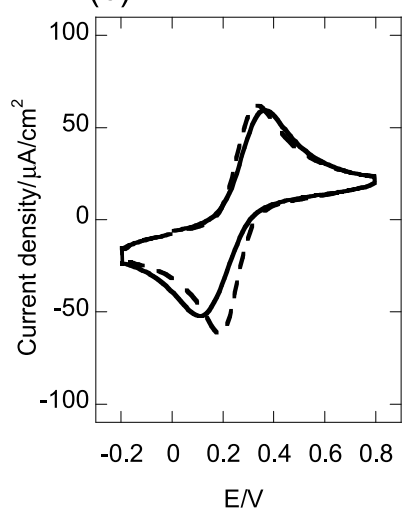

(c)

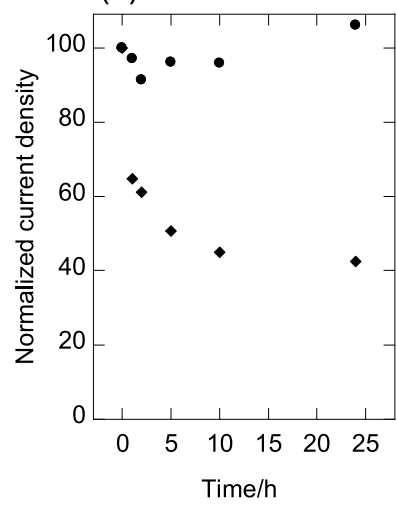

Fig. 4 Cyclic voltammograms for $0.1 \mathrm{mM}$ ferrocyanide obtained on (a) GC and (b) DLC treated with oxygen plasma. Solid curves and dashed curves are before and after treating with $10 \mathrm{mg} / \mathrm{mL}$ DNA for $24 \mathrm{~h}$, respectively. (c) Relationships between the magnitude of the oxidation peak for the $\mathrm{Fe}(\mathrm{CN})_{6}{ }^{4}$ and the time for the DNA treatment on the GC (diamond) and DLC (circle).

DLC surface was remarkably suppressed, as compared with the case of GC. Figure 2 shows AFM images of GC and DLC surfaces before and after treating with $10 \mathrm{mg} / \mathrm{mL}$ BSA for $1 \mathrm{~h}$. Countless valley structures in the image of GC before treating with BSA began to disappear, and became broad after the BSA treatment (Figs. 2a and 2b), while there was no significant change in the image of the DLC before and after (Figs. 2c and $2 \mathrm{~d})$. Moreover, the calculated surface roughness $\left(R_{\mathrm{a}}\right)$ values increased due to the BSA treatment from 1.5 to $5.3 \mathrm{~nm}$ at GC and from 1.0 to $1.7 \mathrm{~nm}$ at DLC, indicating that the surface roughness of the GC remarkably increased compared to the DLC. The fluorescence from each plate surface was also measured to estimate the adsorption of BSA. The surfaces were treated with a phosphate buffer solution containing fluorescein-attached BSA $(1.0 \mathrm{mg} / \mathrm{mL})$ through the PDMS channel for $1 \mathrm{~h}$, and then washed with a phosphate buffer. The fluorescence reflected the channel form was clearly observed from the GC surface, but no significant signal from the DLC surface (Fig. 3). These results also give further evidence for this anti-fouling ability. 
On the other hand, the current decrease for the $\mathrm{Fe}(\mathrm{CN})_{6}{ }^{4-13-}$ system on the GC is considered to be caused by electrostatic repulsion between the anionically-charged BSA $(\mathrm{pI}=4.9)$ adsorbed on the electrode surface and the redox species in the buffer solution. When we used the ferrocene/ferricinium-redox couple or the $\mathrm{Ru}(\mathrm{II}) / \mathrm{Ru}(\mathrm{III})$-complex as a redox marker, no significant current decrease was observed on the GC, even after soaking in the BSA solution. This shows that the anionic redox marker is far more sensitive to detect electrode fouling by BSA and other anionic macromolecules, such as human serum albumin (HSA, $\mathrm{pI}=4.8$ )-containg sera and DNA.

Effect of DNA and human sera on the redox responses for $\mathrm{Fe}(\mathrm{CN})_{6}^{4-/ 3-}$

Figures $4 \mathrm{a}$ and $4 \mathrm{~b}$ show the $\mathrm{CVs}$ for the $\mathrm{Fe}(\mathrm{CN})_{6}{ }^{4-13-}$ system on the GC and DLC electrodes, before and after soaking each electrode in $10 \mathrm{mg} / \mathrm{mL}$ DNA (from herring sperm), respectively. The current responses for the redox system on the GC electrode decreased after soaking it in the DNA solution, and the redox peak currents became $c a .40 \%$ of the initial value (i.e., the resoponses before soaking the electrode in the DNA solution) after soaking the electrode for $24 \mathrm{~h}$ (diamonds in Fig. 4c). In contrast, soaking the DLC electrode did not cause any significant effect on the redox properties of the electrode for the $\mathrm{Fe}(\mathrm{CN})_{6}{ }^{4-13-}$ system (circles in Fig. 4c).

The treatment of the DLC in diluted sera (the dilution ratio, from $1 / 100$ to $1 / 10$ ) also did not show any significant effect on the electrochemistry of the $\mathrm{Fe}(\mathrm{CN})_{6}{ }^{4-13-}$ system, although that of $\mathrm{GC}$ brought about a significant decrease in the redox current for the same species.

Thus, the DLC electrode has been proved to exhibit a high anti-fouling ability in solutions containing BSA, human serum and DNA. The anti-fouling ability leads us to construct DLC-based biosensing systems. Such an investigation is now under progress.

\section{Acknowledgements}

The authors are grateful to Prof. Takashi Osumi and Prof.
Fumiko Hirose at University of Hyogo for their help in fluorescence measurements, and to Mr. Makoto Okada, who is $\mathrm{Ph}$. D. candidate at University of Hyogo in AFM measurements. This work was partly supported by a Grant-in-Aid for Scientific Research (No. 22655022) from the Ministry of Education, Culture, Sports, Science and Technology of Japan.

\section{References}

1. R. L. McCreery, Chem. Rev., 2008, 44, 2646.

2. R. Hauert, Diamond Relat. Mater., 2003, 12, 583.

3. V. Rehacek, L. Hotovy, and M. Voys, Sens. Actuators, B, 2007, 127, 193.

4. G. C. Fiaccabrino, X.-M. Tang, N. Skinner, N. R. Rooji, and M. Koudelka-Hep, Anal. Chim. Acta, 1996, 326, 155.

5. R. Schnupp, R. Kuehnhold, G. Temmel, E. Brurte, and H. Ryssel, Biosens. Bioelectron., 1998, 13, 889.

6. J. Kim, A. Bordeanu, and J.-C. Pyun, Biosens. Bioelectron., 2009, 24, 1394.

7. O. Niwa, J. Jia, Y. Sato, D. Kato, R. Kurita, K. Maruyama, K. Suzuki, and S. Hirono, J. Am. Chem. Soc., 2006, 128, 7144.

8. N. Sekioka, D. Kato, R. Kurita, S. Hirono, and O. Niwa, Sens. Actuators, B, 2008, 129, 442.

9. K. Goto, D. Kato, N. Sekioka, A. Ueda, S. Hirono, and O. Niwa, Anal. Biochem., 2010, 405, 59.

10. A. Fujishima, Y. Einaga, T. N. Rao, and D. A. Tryk, "Diamond Electrochemistry", 2005, Elsevier B. V., Amsterdam.

11. J. Wang and P. Tuzhi, Anal. Chem., 1986, 58, 1787.

12. N. Sekioka, D. Kato, A. Ueda, T. Kamata, R. Kurita, S. Umemura, S. Hirono, and O. Niwa, Carbon, 2008, 46, 1918.

13. M. G. Granger, J. Xu, J. W. Strojek, and G. M. Swain, Anal. Chim. Acta, 1999, 397, 145. 